\title{
INDEX OF NAMES*
}

Abel, W., 459

Abella, I., 268

Abella, R., 134

Abendroth, W., 287

Abensour, M., 122

Abou, A., 447

Abrams, Ph., 460

Abrams, Sh., 460

Acquaviva, S. S., 294

L'Action politique des ouvriers québécois, 441

Agnoli, J., 273

Agosti, A., 111f.

Ahrweiler, G., 432

Albeda, W., 134

Albers, F.-J., 108

Allen, W. Sh., 113

Allyn, G., 279

Amann, P. H., 123

The American Revolution, 268

America's Revolutionary Heritage, 44lf.

Anderson, G., 287

Anderson, W. A., 117

Annali [della] Fondazione Letio e Lisli Basso - Issoco, I, 112

Apitzsch, U., 432f.

The Arab-Israeli Conflict, 118

Archiv für Sozialgeschichte, XV-XVI, 451

Arnold, E., 439

Arnot, R. P., 460

Arnould, R., 279

Artelt, W., 125

Arvon, H., 108

Ashtor, E., 443

Aston, M., 292

Atheismus in der Diskussion, 108

Aubin, H., 459

Authier, D., 459
Bader, V.-M., 109f.

Badie, B., 446

Bahne, S., 280

Bain, G. S., 433

$B a k, J ., 459$

Barclay, W., 258

Barkin, S., 265

Barrot, J., 459

Basu, A., 274

Bater, J. H., 135

Batstone, E., 133

Bauchard, Ph., 447

Bay, E. G., 265f.

Bebel, A., 451

Bédarida, F., 437

Beer, S. H., 132

Behn, B., 283

Behn, W., 283

Behnen, M., 452

Beier, G., 282

Bellamy, J. M., 460

Bennett, V. P., 440

Benseler, F., 466

Benson, J., 287f.

Bercé, Y.-M., 277

Berger, J., $109 \mathrm{f}$.

Bergmann, J., 437

Bernal, M., 118

Bernstein, E., 280

Berque, A., 444

Bers, G., 124

Berthe, M., 121

Bertsch, G. K., $259 \mathrm{f}$.

Bibliografia del Socialismo e del Brunbauer, W., vide Rettich, E.

Movimento Operaio Italiano, Brusatti, A., 459

II, Supplemento 1953-1967. Büsch, O., 262, 458 294

Bibliography of British History I85I-1914, 293

Biraben, J.-N., $435 f$.

Blackey, R., 260
Blasius, D., 451

Bleichröder, G. von, 452

Bley, H., 262

Blumé, D., 448f.

Blumhardt, Ch., 439

Bois, G., 447

Boll, F., 260f.

Bonnet, J.-C.. 122

Borchardt, K., 459

Bosl, K., 459

Botschafter Paul Graf von Hatzfeldt, 452

Botz, G., 275

Bourderon, R., 448f.

Bouvier, B. W., 451

Bowen, P., 129f.

Bowman, F.-P., 11]

Brack, R., 452

Brain, J. L., 266

Brandes, S. B., $268 \mathrm{f}$.

Brandt, P., 280

Brannen, P., 133

Braudel, F., 439f.

Brauns, H., 280f.

Breteau, J., 279

Briggs, A., 46If.

Bromley, J. S., 463

Brooks, G. E., Jr, 266

Broom, J., 288

Brownlee, M. M., 442

Brownlee, W. E., 442

Brüggemeier, F., 451

Brugger, W., 273f.

Burgess, K., $460 \mathrm{f}$.

Burles, J., $448 \mathrm{f}$.

Burnett. J., 125

Burridge, T. D., 133

Bushnaq. I., 120

* References to publications listed under the heading of Other Books are printed in italics. 
Calhoun, D. F., 436

Calkins, K. R., 452

Calvet, L.-J., 256

Carmichael, J., 135

Carrère d'Encausse. H., 264

Carroll, B. A., 437

Cartwright, F. F., 461

Castoriadis, C.. 109

Changing Social Structure in Ghana, 115

Chataignier, A.. 447

Chaussinand-Nogaret, G., 277, Ebel, G., 452 447

Chester, N., 130

Chirot, D., 467

Chomsky, N., 120

Christ, K., 452f.

Cliff, T., 133

Cole, G. D. H., 462

Cole, J., 264

Cole, M., 462

Collier, P., 269

Contamine, Ph., 263

Conte, F. 112

Conze, W., 264

Conze, $W ., 459$

Cook, Ch., 464f.

Coomans, P., $295 f$.

Coser, L. A., $258 \mathrm{f}$.

Coste, B., 121

Cournil, P., 447

Crouzet, F., 288

David, M., 447f.

David, P. A., 443

Davis, K., 257

Deacon, A., 462

De Aloysio, F., 466

Degen, G. R., 461

Della Monica, M., 266

Denitch, B. D., 469

Des tracts en mai 68,278

Desfosses, H., 440

Desroche, H., 433

Desrosiers, R., 267f.

Deutsche Amerikaauswanderung im 19. Jahrhundert, $124 f$.

Der Deutsche Bauernkrieg 1524-1526, 281

Dewar, H., 461

De Weerdt, D., 446

Didsbury, B., 464

Diederich, N., 287

Dietrich, B., 287

Dilcher, G., 129

Documents of the History of the Communist Party of India, VII, 119
Dontenwill, S., 122

Douglass, D., 464

Drenkhahn, R., 266f.

Droz, J., 437

Dubois, P., 433

Duby, G., 263

Duncan, W. R., 440

Dupuigrenet-Desroussilles, G.. 111

Dynes, R. R., 117

Eckert, E., 439

Egret, J., 448

Ehni, H.-P., 281

Eley, G., 262

Elliger, W., 125

Eloni, Y., 282

Elsheikh, F., 433

L'Emigration russe en Europe, 297

Enciclopedia dell'antifascismo e della Resistenza, III, 294

Engelhardt, U., 264

Engels, F., 110, $127 \mathrm{f}$.

Engelsing, R., 125

Ernährung- und Ernährungslehre im 19. Jahrhundert, 125

Essays in Canadian Working Class History, 116

Essays in Labour History 1918-1939, 461f.

Estier, R., 122

Eulner, H.-H., 125

Evans, G. E., 130

Everitt, A., 465

Ewing, W. C., 440f.

Faber, B. L., 259

Fanon, F., 258

Fatchett, D., 133

Felzenzwalbe, N., 447

Fesser, G., 453

Fetscher, I., 260, 433

Feuerwerker, D., 448

Field, F., 121

Filler, L., 269f.

Finley, M. I., 439

Finzi, E., 133

Firnberg, H., 276

Fischer, W., 262

Florinsky, M. T., 260

Focke, H., 124f.

Foner, E., 268, 270

Fonnes, I., 436

Foot, M. R. D., 436

Forester, T., 130

Form, W. H., 259

Foschepoth, J., 28 If.
Fournier, J., 122

Franz, G., 125f.

Fraser, D., 462

Frêche, G., 122

Frecot, J., 129

Freudenberg, G., 260

Frieden, Gewalt, Sozialismus, $260 f$.

Friedlander, P., 270

Fritze, W. H., 275

Fuchs, N., 287

Führungskräfte der Wirtschaft, I-II, 453

Fundamentals of the Chinese Communist Party, 274

Gabriel, P., 296

Gager, J. G., $112 \mathrm{f}$.

Galimard, M.-C., 447

Ganschow, Th. W., 259 f.

Ganssmann, H., 109f.

Gauchet, M., 122

Gay, P., 282

Geiss, 1., 261f.

Geldolf, W., 446

Geoffroy, M., 447

Geras, N., 109

The German Peasant War of 1525,459

Gerth, H. H., 126

Gessner, D., 453

Gestri, L., 466

Giessen, K.•H., 126

Goodman, J. F. B., 265

Goody, J., 115

Gore, P., 460

Gorter, H., 459

Goujon, P., 122

Gray, G. B. D., $287 f$.

Gray, R. Q., 130 f.

Great Britain and Her World. 1750-1914, 288

Greenwood, D. J., 296

Gregg, P., 462

Griffin, P. E., 274

Grugel, L. E., 288

Grund, H., 454

Grundfragen der geschichtlichen Beziehungen zwischen Deutschen, Polaben und Polen, 275

Grunenberg, A., 466

Gurr, T. R., 450

Gutman, H. G., 443

Habermas, J., 433f.

Haferkorn, F., 126

Hafkin, N. J., 265f.

Hahn, K., 278 
Hahn, M., 123, 261

Hallgarten, G. W. F., 262

Hallern, U., 459

Hamer, C., 294

Hampe, P., 129, 434

Hanak, T., 293f.

Handbuch der deutschen Wirtschafts- und Sozialgeschichte, 459

Hanham, H. J., 293

Hansen, $\mathrm{Ch}$., $124 \mathrm{f}$.

Harris, A., 124

Harris, B., 465

Harris, J. R., 288

Harrisson, T., 288f.

Hart, K., 115

Hart, P. P. van 't, vide Perthus, $M$.

Hartmann, W.-R., 451

Hatzfeldt, P. von, 452

Haupt, G., 282

Hayden, D., 442

Heischkel-Artelt, E., 125

Heit, A., 448

Hejzlar, Z., 277

Helbig, H., 453

Henderson, W. O., 126f., 288

Hen-Tov, J., 273

Herkunft und Mandat, 282

Herod, Ch. C., 113

Héroux, D., 267f.

Herrmann, U., 126

Herzfeld, H., 262

Hill, F., 440

Hill, P., 115

Hill, S., 462

Hilton, R. H., 292

Hindels, J., 275f.

Hirsch, H., 280, 454

Histoire des paysans français du

XVIIIe siècle à nos jours, 122

Hoelz, M., 45I

Hoerder, D., 437

Hörning, K. H., $256 f$.

Hoffmann, S., 450

Holstein, F. von, 452

Holton, B., 289

Holz, H. H., 432

Hommes et bêtes, 261

Hook, S., 257

Hopkinson, G. G., 287

Horn, P., 462f.

Horowitz, D., 269

Horský, V., 261

Hostetler, J. A., 116

Houssel, J.-P., 122

Howe, I., $270 \mathrm{f}$.

Huber, W., 260f.

Humbert-Droz, Jenny, 261
Hunter, B., 265

Huysmans, C., 446

Hyams, Ph., 468

Hyman, A., 459

Imhof, A. E., 436

Imperialismus im 20. Jahrhundert, $261 f$.

Indian Women, 274

Indian Women, 274 päische Wirtschaft" im 19. Krasucki, H., 279 Jahrhundert, 262 Kraus, J., 440

The Infidel Tradition from Pai- Kriegel, A., 278, 446 ne to Bradlaugh, 289

Internationales Archiv für Sozialgeschichte der deutschen Literatur, 1-2, 454

Ismael, T. Y., 273

Istoričeskij opyt bor'by KPSS Kullack, I,, 287 protiv trockizma, 298

Jäger, G., 454

Jaffe, Ph. J., 117

Jahrbuch der historischen Forschung in der Bundesrepublik Deutschland, 1975, 262 Lamennais, F. R. de, 122

200 Jahre amerikanische Revo- Landauer, G., 459 lution und moderne Revolu- Landes, D. S., 262 tionsforschung, 437

James, L., 292

Jeanneney, J.-N., 449

Jemnitz, J., 264

Jersch-Wenzel, S., 262

Jiryis, S., 120

Jochmann, W., 282

Johnson, Ch. H., 123

Johnstone, F. A., 115

Joll, J., 114

Jones, D., 131

Jones, M., 464

Jones, $\mathrm{Ph} ., 464 f$.

Jonge, T. de, $295 f$.

Jordan, M. V., 268

Jordan, W. C., 438

Josephson, E. F., 467f.

uden im Wilhelminischen Lenin, V. I., 460 Deutschland 1890-1914, Léonard, P., 122 282

Judt, T., 278

Jüdisches Leben in Deutsch- Lévesque, J., 440 land, 282

Jurquet, J., $262 f$.

Kalivoda, R., 121

Kaplan, S. L., 449

Kapp, Y., 289

Kater, M. H., 263, 264

Kealey, G. S., 116

Keep, J. L. H., 297
Leroux, P., 122

Leuschner, J., 129

Lane, A. J., 437

Lane, D., 257

Langewiesche, D., 451

Laqueur, Th. W., 463

La Roque, G. A. de, 277

Larsen, Ø., 436

Larsen, O. N., $258 f$.

Laski, K. 110

, $H, 459$

Lee, A. J., 290

Leech-Anspach, G., 297

Lefebvre, G., 277

Lefort, C., 122

Lefranc, $G$., 449

Lehmann, H. G., 283

Lemerle, P., 263

Levra, U., 466

Lewin, M., 135

Lhomme, J., 277

Liang, Hsi-huey, 283

Liberating Women's History, 437

La Libération de la France, $449 f$.

Libo, K., $270 f$. 
Lieberthal, K., 443f.

Liebknecht, W., 451

Linhart, S., 444

Link, W., 282

Link-Salinger (Hyman), R., 459

Linse, U., 129

Lipgens, W., 264

Lovell, J., 462

Lucas, E., 455

Ludwig, M. H., 455

Ludz, P. Ch., 110

Lübbe, H., 108

Luthra, B., 274

Lutz, B., 127

McCulloch, A., 460

Macfarlane, J., 288

McKee, J. B., 258

MacLaren, A. A., 132

McLeod, D. J., 271

McNab, B., 438

McPherson, J. M., 271

März, E., 110

Mahon, J., 131

Maihofer, W., 108

Maimann, H., 120

Maione, G., $294 f$.

Maitron, J., 437

Mamdani, M., $115 f$.

Mandel, A., 452

Manning, B., 290

The Manning of the Royal Navy, 463

Manton, J., 291

Markov, W., 123

Martinez-Alier, J., 441

Martino, A., 454

Martiny, M., 283

Marx, K., 110, 127f.

Marx-Lexikon zur politischen Okonomie, 6-9, 110

Mason, T. W., 455f.

Mazzini e i repubblicani italiani, 295

Mazzini, G., 295

Meek, R. L., 111

Meier, K., $283 \mathrm{f}$.

Mélanges d'histoire sociale offerts à Jean Maitron, 437

Meller, H. E., 291

Mennicke, C., 439

Merkl, P. H., 129

Merli, S., 295

Merton, R. K., 257

Mettler, A., 438

Meyer, A. G., 260

Meyer, F., 456

Meyer, G. P., 437

Michel, B., 445
Middleton, N., 291

Międzynarodowy ruch robotniczy, $437 f$.

Migdal, J. S., 265

Miller, M. A., 438

Milligan, S., 29lf.

Miners, Quarrymen and Saltworkers, $463 \mathrm{f}$.

Misgeld, K., 113

Mittmann, U., 284

Mockenhaupt, H., 280 f.

Möller, D., 438

Mörner, M., 258

Moltmann, G., $124 f$.

Mommsen, H., 263, 264

Mommsen, W. J., 434

Moore, J. N., 118

Morgan, D. W., 456

Morrill, J. S., 464

Mosse, W. E., 282

Le mouvement syndical dans la Résistance, 279

Mullings, L., 266

Musson, A. E., 288

Nagata, J. A., 275

Nanda, B. R., 274

Nash, G. B., 268

Natorp, P., 451

Neumann, F., 456

Neumann, M., 434

Neville, R. G., 287 f.

Niethammer, L., 451

Nijhof, E., 295f.

Nishikawa, M., 262

La Noblesse au Moyen Age, 263

Noel, G., 292

Noll, A., 128

Nolte, E., 113, 263

North, D. C., 262

Novack, G., $441 f$.

Obelkevich, J., 464

Odaka, K., 444f.

Oertzen, P. von, 284

Offenberg, M., 273

Offerlé, M., 447

Oksenberg, M., 443

On Strike, 268

Opdenberg, M.-L., $276 f$.

Order and Innovation in the Middle Ages, 438

Ossorguine-Bakounine, T, 297

Otter, C. von, 265

Overbeek, J., $257 f$.

Owens, L. H., 117

Papa, E. R., 295

The Paris Commune 1871, 279
Parker, J. J., 442f.

Parman, D. L., 118

Parmentola, V., 295

Patterson, D. S., 443

Paucker, A., 282

Payne, H. C., 450

Payne, S. G., 113

Pearse, A., 267

Peasants, Knights and Heretics. 292

Peiró, J., 296

Peper, B., 265

Perels, J., 287

Perrolle, P. M., 274

Perthus, M., 466f.

Peston, M., 258

Petersen, J., 263

Peukert, D., 287

Pfeiffer, A., 438f.

Phan Thien Chau, 445

Pinkney, A., 27lf.

Plotnick, R. D., 272

Pluralism in Malaysia, 275

Poeze, H. A., 119

Poliakov, L., 261

Pollard, S., 262

Polskie programy socjalistyczne 1878-1918, 134

Poster, M., 279

Pouthas, C., 277

Powell, J. E., 258

Price, R., 123

Print and the People 1819-1851, 292

Prümm, K., $456 f$.

Pudal, B., 447

Pulzer, P., 282

Purcal, J. T., 275

Questiaux, N., 122

Rabinowitch, A., 468

Rabočij klass $i$ industrial'noe razvitie SSSR, 298

Racial Conflict, Discrimination, \& Power, 258

Racine, N., 437

Radek, K., 438

Rademacher, G., 284 f.

Radkau, J., $261 f$.

Ragaz, L., 439

Raitz, W., 457

Rama, C. M., 134

Rao, M. B., 119

Ratcliffe, B. M., 288

Ratheau, P., 447

Ratti, G., 295

Reappraisals of Fascism, 113

Regionale und lokale Răteorga- 
nisationen in Württemberg 1918/19, 285

Religiöse Sozialisten, 438f.

Rémond, R., 279

Resler, H., 259

Rettich, E., 434f.

Reuther, V. G.. 272

Revah, M., 447

Revolution and Reaction, 123

Riasanovsky, N. V., 297

Richards, L., 468

Richarz, M.. 282

Riesman, D., 259

Rigler, E., 276

Roberts, P., 115

Robrieux, Ph., 124

Rogalla von Bieberstein, J., 439

Rogozinski, J., 438

Romein, J., 114

Roobol, W. H., 468

Rosen, S., 265

Rossi, P. H., 259

Rotz, Rh. A., 438

Rougerie, J., 437

Rout, L. B., Jr, 267

Royle, E., 289

Ruiz, T. F., 438

Runciman, W. G., 256

Rupieper, H. J., 262

Saage, R., $128 \mathrm{f}$.

Sacks, M. P., 469

Saint-Simon, H. de, 123

Samuel, R., 463f.

Sandys, R., 465

Santuccio, M., 294

Sarkar, L., 274

Sass, H.-M., 108

Sauer, W., 113

Saul, K., 451

Saville, J., 460, $461 f$.

Schäfer, E., $108 f$.

Schäfer, G., 456

Schaeffer, J., 279

Schaff, A., 439

Schenda, R., 263

Schieder, W., 263

Schilling, H., 281, 437

Schissler, J., 260, 435

Schmollinger, H. W., 287

Schönhoven, K., 285, 451

Schofer, L., 459

Schonfeld. W. R., 450

Schram, S. R., 260

Schröder, H.-Ch., 437

Schütze, F., 258

Schulkind, E. W., 279

Schulz, G., 129

Schumacher, M., 285
Schumann, H.-G., 282

Schwarz, K., 457

Schwarz, Ph., 256

Schwerdtfeger, J., 260 f.

Sedgwick, P., 132

Sédouy, A. de, 124

Sellin, V., 264

Seminar: Die Entstehung der antiken Klassengesellschaft, 439

Sengle, F., 454

Sexsmith, A., 465

Seyd, P., 462

Shapiro, Y., 120

Shipley, P., 464

Shostak, A. B., 259

Sieburg, H.-O., 123 f.

Sieyès, E. J., 277

Sigel, R., 457

Silberner, E., $285 f$.

Silver, H., 131

Simms, R. P., 258

Sinclair, J., 464f.

Sismondi Européen, 111

Sivan, E., 266

Skidelsky, R., 293

Skidmore, F., 272

Skrzypczak, H., 282

Slater, Ph., 435

Slaven, A., 292f.

Smout, T. C., 132

Snyder, L. L., 458

Social Class in Scotland, 132 Valiani, L., 295

The Social Structure of Eastern Veenhoven, W. A., 440f. Europe, 259

Socialism in the Third World, Vitale, L., 116 440

Sontheimer, K., 458

Sources in British Political His- Vucinich, A., 469 tory 1900-1951, 2-4, 464f.

Soziale Bewegung und politische Verfassung, 264

Spiers, J., 465

Spitaels, G., 276

Stachura, P. D., 129

Stadler, K. R., 445

Stalnaker, J. C., 281

Stampp, K. M., 443

Stearns, P. N., 450

Steinbach, P., 458

Stelling-Michaud, S., 111

Stephanidès, M., 447

Stephenson, J., 286

Stinchcombe, A. L., 259

Stoianovich, T., 439 f.

Strutynski, P., 458f.

Stürner, W., 114

Stuke, H., 264

Sucharowski, W., 286
Vondung, K., 129

Vos, H. de, 467

Sutch, R., 443

Taylor, K., 123

Temin, P., 443

Tholfsen, T. R.

Thompson, E. P., 132

Tillich, P., 439

Tilton, T. A., 286

inker, H., 440

Tecqueville, A, de, 277

Topolski, J., 440

Trautmann, G., 451

d, L., 111

Tudesq, A.-J., 277

Turner, H. A., Jr, 113

Ulam, A. B., $135 f$.

Ullmann, H.-P., 459

Umbreit, H., 450

The Uses of Controversy in Sociology, $258 \mathrm{f}$.

Wahlstatistik in Deutschland,

Wang, J. C. F., 119

Warrian, P., 116

Webb, B., 132

Webb, S., 132

Weber, E., 124

Weeks, J., 464f.

Wehler, H.-U., 281, 437

Wehler, H.-U., 459

Weill, C., 446

Weissel, E., 276

Weitzman, S., 291

Westergaard, J., 259

Wetzel, H.-W., 287

White, Ph., 133

Widgery, D., 132f.

Wiegelmann, G., 125

Das wilhelminische Bildungs- 
bürgertum, 129

Wilkinson, F., 462

Will, E., 439

Wilson, A. J., 440

Winkler, H. A., 263

Wistrich, R. S., 114

Wölk, M., 262

Wölk, W., 262

Wohlfeil, R., 281

Wojtasiewicz, O., 440

Wolfe, B. D., 117

Wolffheim, F., 459

Wolpe, H., 258

Women in Africa, 265f.

Woodhouse, C. M., 133

Woodroofe, D., 442

The Worker Directors, 133

Worker Militancy and Its Consequences, 1965-75, 265

Wright, Gavin, 443

Wright, J. C., 442

Wright, V., 123

Yates, G. G., 272

Young, A. F., 268

Zaninovich, M. G., 260

Zernack, K., 275

Zoll, R., 111

Zorn, W., 459 